\title{
An Efficient Method For Extracting The Necessary Features In A Multimodal Biometrics
}

\author{
S.Arunarani, R.Gobinath
}

\begin{abstract}
Identifying the person using the unique textures of face, ear and fingerprint can be extracted very swiftly during the recognition process. Preprocessing the acquired image and extracting the necessary features from the preprocessed image are very important for any image based applications. The success of the process is entirely depending on the conflux rate and efficiency of such techniques. But, because of the negligence of the implication of these techniques results in the failure of the process. In this paper, appropriate pre-processing techniques are identified to extract the necessary features from the images for better performance.
\end{abstract}

Keywords: Feature extraction, images of face, ear, and fingerprint, Pre-processing,.

\section{INTRODUCTION}

In biometrics, identification of a person is done through the physiological and behavioral characteristics of a human. Thus, human features are used in many areas where security is needed. However, single trait of a human has various disadvantages such as permanence of the trait, spoof attack, peculiarity of the trait etc,. To overcome these disadvantages of unimodal biometrics, more than one trait is used for identification of a human [4]. In multimodal biometrics, more than one trait of the human is used for identifying the individual person. The main issue in multimodal biometrics lies in combining multiple features from each trait to create good recognition of a human.

The most advanced and approved biometric system is the fingerprint recognition system. Fingerprint recognition means identifying equivalent prints of human thumb. Fingerprint recognition systems are accepted universally because of its uniqueness in different people. Fingerprint is easily attainable feature of biometrics, which is reliable and fool proof for the identification of human beings.

Face recognition is submissive and non-protruding, different from other dynamic biometric techniques such as those using fingerprints, speech and signature. Ear is a new feature of human biometrics for identification with exclusivity and durability. Ear has data affluent anatomical characteristics and unaltered by ageing. Its position on the surface of the head makes extraction easier.

Revised Manuscript Received on July 05, 2019.

Mrs.S.Arunarani*, Assistant professor, Prince Shri Venkateshwara Arts \& Science College, Research Scholar, Department of computer science, VISTAS, Chennai, India.

Email: sarunaarani@yahoo.co.in.

Dr.R.Gobinath,Associate Professor, Department of computer science,

VISTAS, Chennai, India.

Email: iamgobinathmca@gmail.com.
Normally, pictures possessed from scanning and virtual images composed from candidly obtainable list are used for picture categorization and partitioning. These are unrefined pictures which are inappropriate for scrutiny owed to the various categories of noises currently in the pictures. Therefore, proper preprocessing techniques must be utilized to improve the eminence of the pictures.

In diverse computer visualization applications broadly utilized is the progression of recouping preferred pictures from a huge compilation on the base of characteristics that can be routinely derived from the pictures themselves. These systems are called Content Based Image Retrieval. CBIR have acquired severe concentration in the text of picture data reclamation. In view of the fact that this part was on track years ago, and as a result, an extensive variety of strategies has been projected. The methods utilized in these structures are generally branched into three functions: - distillation, selection, and - categorization. The distillation function converts the thriving composition of pictures into different features. Feature extraction is the procedure of producing features to be utilized in the selection and categorization tasks. Feature selection diminishes the amount of features applied to the categorization function. Those characteristics which are expected to support in bias are preferred and used in the categorization function. Features which are not preferred are rejected [1].

Following phases are present in the process:

- Preprocessing: In this stage the image is refined in order to extort the textures, which express its filling. The processing incorporates refining, standardization, partitioning, and object recognition. The yield of this phase is a collection of momentous domains and substances.

- Feature extraction: To explain the substances of the images, characteristics such as outline, consistency, color, etc. are utilized. Image characteristics can be grouped into primitives.

\section{LITERATURE REVIEW}

Jing Zhang et al., proposes a multimodal biometric recognition approach using faces and fingerprints. In their paper LBP is used for recognizing more features and combines the extracted features into a single bundle. It also utilize the robust probabilistic collaborative representation based classifier for recognizing the person.[5]

Chang et al., initiate a multimodal system based on face and ear. PCA is utilized to extort the feature and feature level fusion is

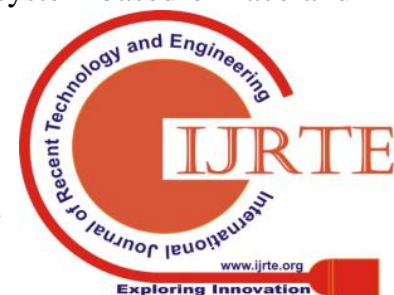




\section{AN EFFICIENT METHOD FOR EXTRACTING THE NECESSARY FEATURES IN A MULTIMODAL BIOMETRICS}

used for concatenation problem.[6].

In their paper Pan et al. [7] suggested kernel principal component analysis (KPCA) but with lack of recognition rate $94.5 \%$.

Mahoor et al. [8] used 3D ear images and 2D face images. Active shape model and Gabor filters are used to extract from face images. Shape from shading algorithm was used to obtain the $3 \mathrm{D}$ ear images. Score level fusion is used to achieve higher recognition rate. But $3 \mathrm{D}$ ear recognition system is very expensive which limits its usage.

Li et al. [10] proposed a method in which 2D Fisher linear discriminant is used for feature extraction to attain better recognition rates using face and ear traits.

\section{PROPOSED METHOD}

The method proposed here consists of three steps (Fig.1).

1. Image acquisition of the user from sensor.

2. Preprocessing operations to develop the superiority of the image.

3. Feature extraction to get the necessary features for identification.

After the feature extraction, matching is done using the extracted feature and the images in the database. Then decision is analyzed for recognition. Amperes and magnetic field in ousted. This repeatedly passes to mystification because equations do not poise dimensionality.

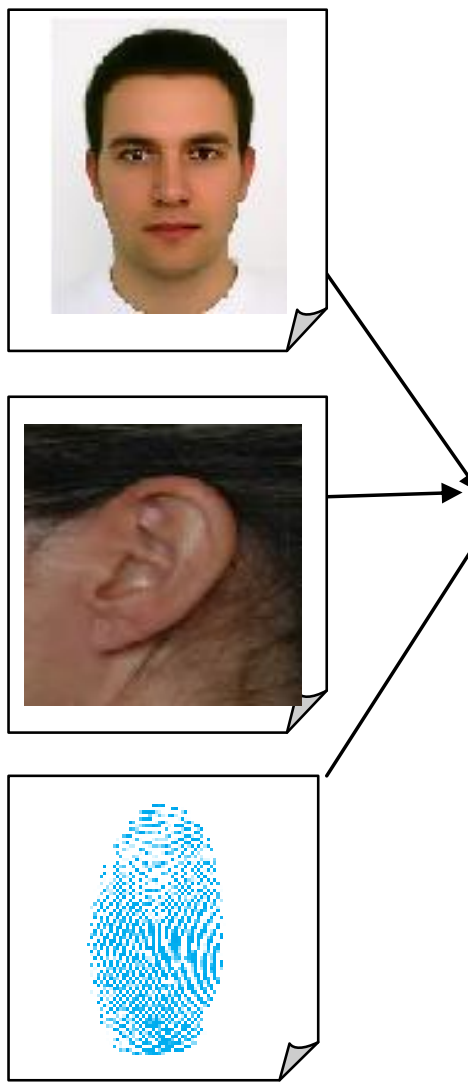

Fig 1: Proposed Method

\section{IMAGE ACQUISITION}

Acquisition of a facial image can be acquired through a digital camera to capture a live picture of a face or a scanned photograph of the person. Algorithms like LDA can recognize faces with different illumination and because of this different illumination is not an issue.

Acquisition of an ear image can be acquired through a digital camera to capture a live picture of a subject's ear or a scanned photograph of the person's ear. Acquisition of a finger print image is acquired from an optical fingerprint sensor. It is 
developed such that it works in all operational conditions to provide strong spoof detection.

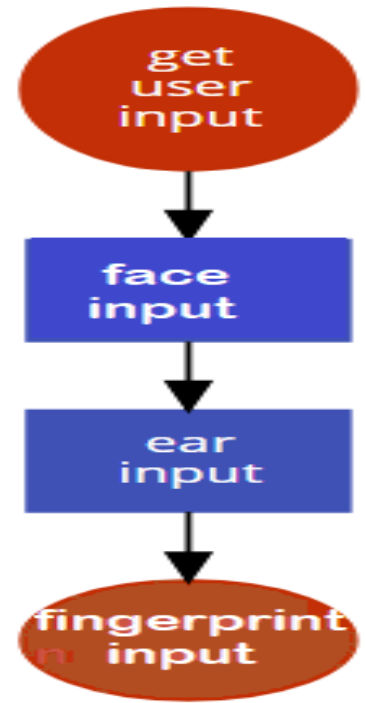

Fig.2: Image Acquisition

\section{IMAGE PREPROCESSING}

Image refining can widely boost the reliability of a visual examination. Various filtrate techniques are used to enhance or reduce the information of the image. Users can enhance a camera picture with just a small amount of snaps. Image preprocessing diminishes executing instant and amplifies the probability of exact matching [5]. Face, fingerprint and ear images are preprocessed to appease the necessary conditions for feature extraction.

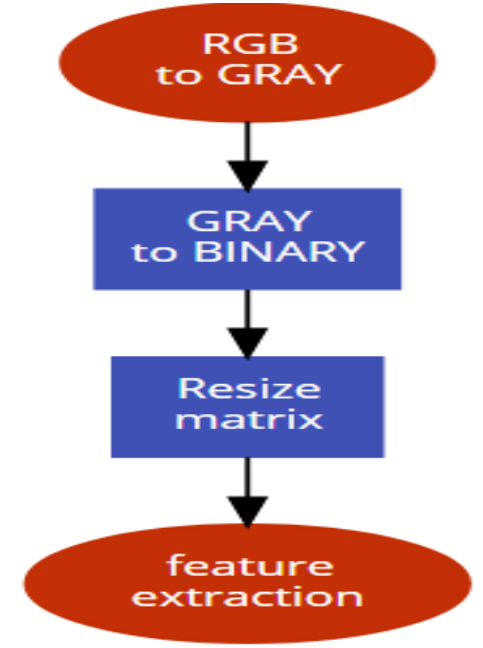

Fig.3: Preprocessing

\section{FEATURE EXTRACTION}

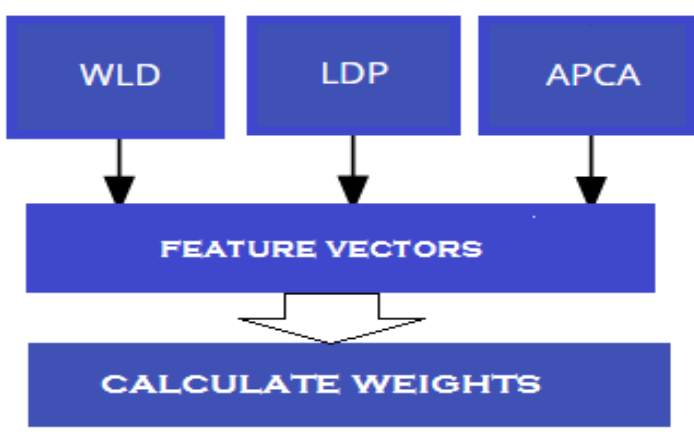

Fig.4: Feature Extraction

\section{A. Extraction Local Feature Descriptors}

Computer vision algorithms use local features and their descriptors as their edifice blocks. Some of the implementations are picture enrolling, object revealing and classification, capturing and motion approximation. Local features are used by these algorithms to manage range changes, revolution, and occlusion. The Computer visualization System Toolbox ${ }^{\mathrm{TM}}$ accommodate the FAST, Harris, and Shi \& Tomasi procedures for detecting curve characteristics, and the SURF, KAZE, and MSER approaches for detecting blob characteristics. The toolbox comprises of the SURF, KAZE, FREAK, BRISK, and HOG descriptors. Based on the necessities the detectors and the descriptors can be merged.

\section{B. Weber Local descriptor}

In this paper we recommend a straightforward, still extremely influential and forceful local descriptor, called the Weber Local Descriptor (WLD) [17].WLD suggest that human inkling of an outline depends on the adjustment of a catalyst (such as resonance, illumination) and also on the innovative strength of the catalyst. WLD comprises of two factors: differential excitation and orientation. The differential excitation segment is the fraction amidst the relative power distinctness of a present pixel against its neighbors and the force of the existing pixel. The orientation segment is the incline orientation of the present pixel [17].

\section{Adaptive PCA}

To solve recognition problems, dimensionality reduction can be used. This is known as Principal component analysis (PCA). It is also identified as Eigen space projection, Karhunen and Loeve (KL) alteration, or Hotelling. PCA condense amplitude by withdrawing the Principal factors of multidimensional information. PCA can obtain the vital characteristics, record the practically erratic data parts of samplings, and then opt for abundant momentous entities from every one of the characteristics factors. Through PCA, a competent and uncomplicated identification process can be attained correlated with other methods. Recognitions that employ PCA characteristics also execute finer in particular deviation cases for every individual. Unrefined strength data are used for identification and knowledge accusation without middle level or lower level processing. PCA is unverified and does not need any acquaintance about face pictures. Data condensation can also be estimated using the subspace of the low-dimensional depiction. In face identification the accomplishment of face identification called Eigen face technique.

The phases in Eigen face technique are as follow [18]:

1. Depict images as column angle.

2. The intermediate mean for the training pictures is calculated using the following equation:

$$
\text { Average Mean }=\frac{1}{M} \sum_{n=1}^{M} \text { TrainImage }(n)(1)
$$

Where $\mathrm{M}$ is the total number of training pictures,

3 . The difference between standard image from the calculated image is calculated as follows:

$S=$ TrainImage

AverageMean (2) 


\section{AN EFFICIENT METHOD FOR EXTRACTING THE NECESSARY FEATURES IN A MULTIMODAL BIOMETRICS}

4. The covariance matrix is determined as follows :

$$
\text { Covariance }=\frac{1}{M} \sum_{n=1}^{M} S(n) S^{T}(n)
$$

7. Visualize the preparation instance onto the Eigen faces.

VII. RESULT AND DISCUSSION

5. Eigen values and Eigenvectors need to be estimated.

6. Arrange and eradicate Eigen values.

TABLE 1: PREPROCESSING RESULTS

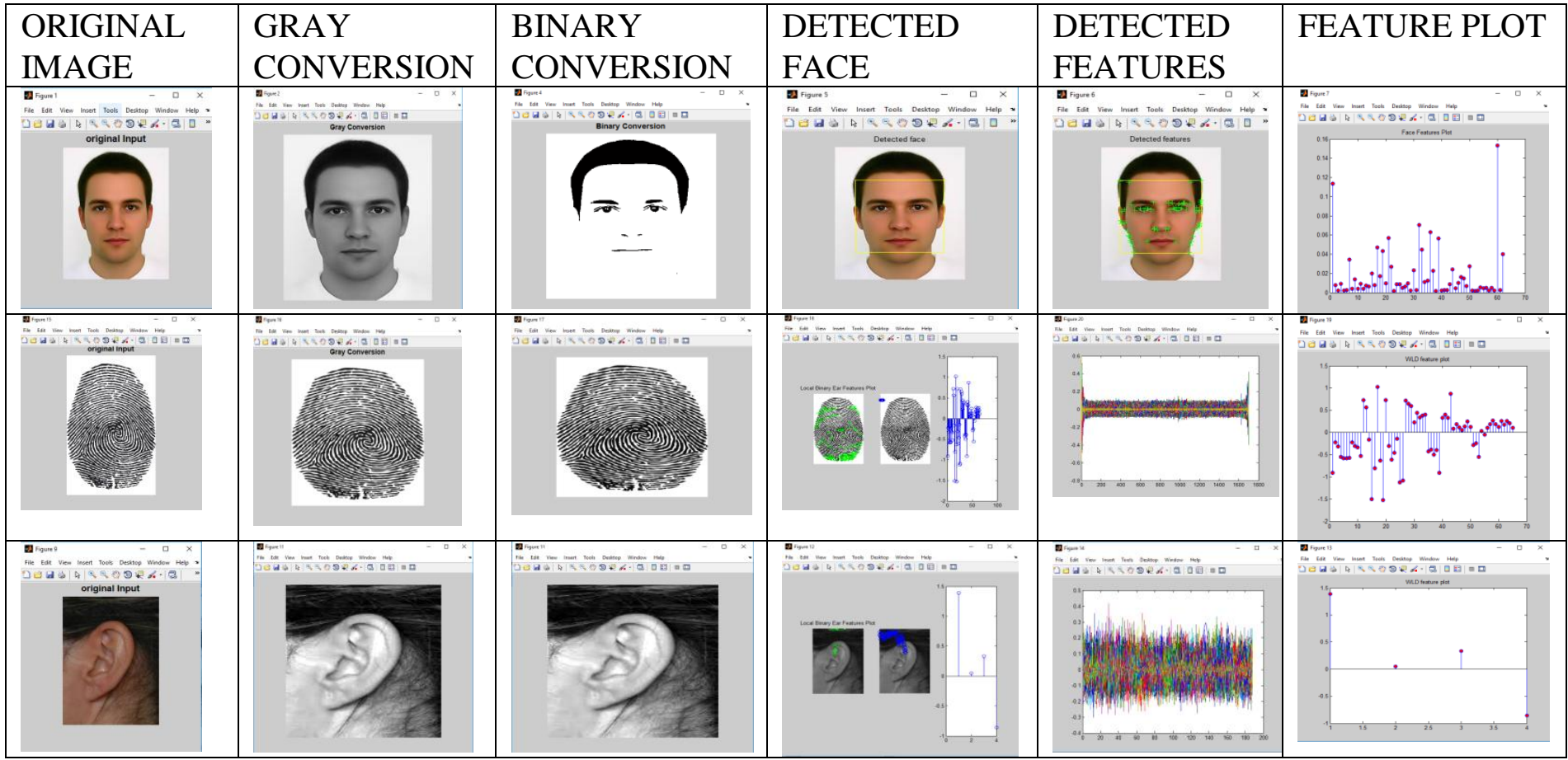

This segment demonstrates the experimental results of a multimodal systems and the projected fusion stratagem on diverse traits. There is no existing face - fingerprint - ear multimodal database gathering face, ear and fingerprint images of the corresponding subject. Thus, the entire analysis is carried away on an implicit multimodal database linking face, ear and fingerprint from three autonomous unimodal databases.

To assess the presentation of the planned routine against the obtainable state-of-the-art routines, operations are carried out over the similar databases.

An equivalent output is attained in evaluation with the advanced systems as shown in Table.2. The attainment of our projected system is appreciably better than some of the projected multimodal systems. The following table gives the evaluation of the average time and memory usage for the four feature extraction techniques. PCA and WLD had the fastest matching speed with an average time of $0.63 \mathrm{~s}$ while SURF had $0.71 \mathrm{~s}$ and Gabor was $0.87 \mathrm{~s}$. The average time for the entire four feature extraction algorithm was $0.72 \mathrm{~s}$.

Fig. $5 \& 6$ depicts the recognition time and mean memory of each Feature Extraction Algorithm.

TABLE 2:

Comparative study between different algorithms

\begin{tabular}{|l|l|l|}
\hline $\begin{array}{l}\text { Name of the } \\
\text { Algorithm }\end{array}$ & Mean Memory (KB) & $\begin{array}{l}\text { Mean } \\
\text { Recognition } \\
\text { Time (s) }\end{array}$ \\
\hline PCA+WLD & 72.5 & 0.63 \\
\hline PCA & 57.6 & 0.67 \\
\hline Gabor feature & 32.91 & 0.87 \\
\hline SURF & 45.52 & 0.71 \\
\hline
\end{tabular}

\section{Mean Memory (KB)}

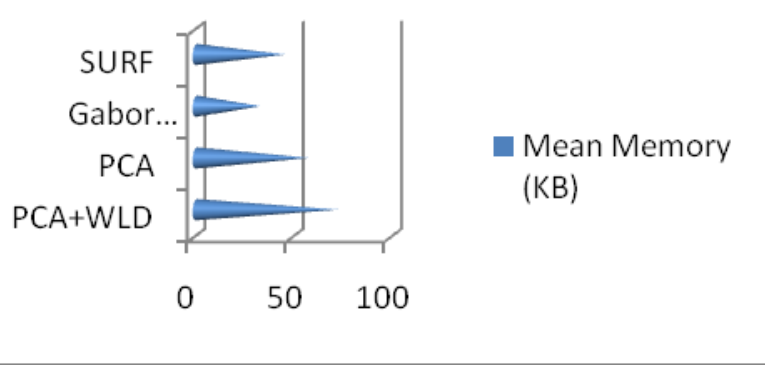

Fig.5:Mean Memory of all the four algorithms 


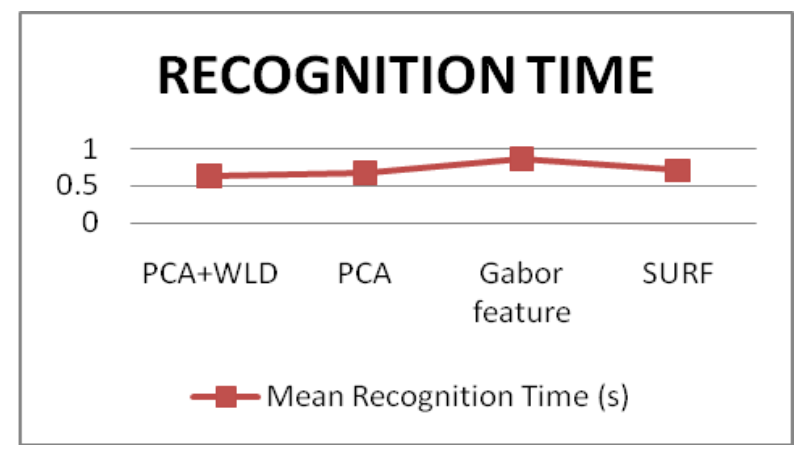

Fig.6:Recognition time of all the four algorithms

\section{REFERENCES}

1. Choras, Ryszard S. "Image feature extraction techniques and their applications for CBIR and biometrics systems." International journal of biology and biomedical engineering 1.1 (2007): 6-16.

2. Jude Hemanth D., Anitha J. (2012) Image Pre-processing and Feature Extraction Techniques for Magnetic Resonance Brain Image Analysis. In: Kim T., Ko D., Vasilakos T., Stoica A., Abawajy J. (eds) Computer Applications for Communication, Networking, and Digital Contents. Communications in Computer and Information Science, vol 350. Springer, Berlin, Heidelberg

3. Jabid, Taskeed \& Hasanul Kabir, Md \& Chae, Oksam. (2010). Local Directional Pattern (LDP) for face recognition. International Journal of Innovative Computing, Information and Control. 8. 329 - 330. 10.1109/ICCE.2010.5418801.

4. Bommagani, Gautam. (2013). Design, Implementation and Evaluation of an Algorithm for Face Recognition Based on Modified Local Directional Pattern. International Journal of engineering research and technology. 2. 2280-2283.

Jing Zhang, Huanxi Liu,Derui Ding, Jianli Xiao, "A robust probabilistic collaborative representation based classification for multimodal biometrics", Proc. SPIE 10615, Ninth International Conference on Graphic and Image Processing (ICGIP 2017), 106151F (10 April 2018).

Chang $K$, Bowyer $K W$, Sarkar S, Victor $B$ Comparison and combination of ear and face images in appearance-based biometrics. IEEE Transactions on pattern analysis and machine intelligence. 2003;25(9):1160-1165.

Pan $X, X u X$, Lu $Y$, Cao $Y$. Feature Fusion in Multimodal Recognition Based on Ear and Profile Face. Proceedings of SPIE - The International Society for Optical Engineering. 2008;1(1):89.

8.

Mahoor MH, Cadavid S, Abdel M. Multi-modal ear and face modeling and recognition. 2009;p. 4137-4140.

Pflug A, Busch C. Ear biometrics: a survey of detection, feature extraction and recognition methods. IET biometrics. 2012;1(2):114-129.

10.

Li Y, Yuan W, Sang H, Li X. Combination recognition of face and ear based on two-dimensional fisher linear discriminant. In: Software Engineering and Service Science (ICSESS), 2013 4th IEEE International Conference on. IEEE; 2013. p. 922-925.

11.

Yuan $L, M u Z C, X u X N$. Multimodal recognition based on face and ear. In: Wavelet Analysis and Pattern Recognition, 2007. ICWAPR'07. International Conference on. vol. 3. IEEE; 2007. p. 1203-1207.

12. Piyush G. Kale, Khandelwal C.S, ' IRIS Finger Print Recognition Using PCA for Multi Modal Biometric System', International

\section{CONCLUSION}

In this paper, for the usage of biometrics in security I have used the method of principal component analysis followed by score generation which yields a good result. It was found that ear, face and fingerprint possesses many of the biometric properties which make it apt for usage in security sensitive areas.

Conference on Global Trends in Engineering, Technology and Management, ISSN 2231-5381, pp. 78-81, 2016

13. Manjunathswamy B E, Dr Thriveni J land, Dr Venugopal $K R$, 'Bimodal Biometric Verification Mechanism using Fingerprint and Face Images(BBVMFF)', 2015 IEEE 10th International Conference on Industrial and Information Systems, Sri Lanka, pp. 372-377, ICIIS 2015.

14. Li B, and Chang T. (2015) Ear Biometric in 2D images Institute of Ear Biometric Research (IEBR) Journal, 15(3). 169-181.

15. Afolabi, Adeolu \& Desmond, Ademiluyi. (2015). Performance Evaluation of Some Selected Feature Extraction Algorithms in Ear Biometrics. International Journal of Science and Research (IJSR). 438.

16. Farmanbar, M. \& Toygar, Ö. Feature selection for the fusion of face and palmprint biometrics https://doi.org/10.1007/s11760-015-0845-6

17. J. Chen et al., "WLD: A Robust Local Image Descriptor," in IEEE Transactions on Pattern Analysis and Machine Intelligence, vol. 32, no. 9, pp. 1705-1720, Sept. 2010.

18. Barnouti, Nawaf Hazim. "Improve face recognition rate using different image pre-Processing Techniques." American Journal of Engineering Research (AJER) 5.4 (2016): 46-53.

19.Ravi, S., and SadiqueNayeem. "A study on face recognition technique based on eigenfacell." International Journal of Applied

Information Systems5, no. 4 (2013): 57-62. 